\title{
AN ORPHAN NO LONGER? DETECTION OF THE SOUTHERN ORPHAN STREAM AND A CANDIDATE PROGENITOR
}

\author{
Carl J. Grillmair ${ }^{1}$, Lauren Hetherington ${ }^{2}$, Raymond G. Carlberg $^{2}$, and Beth Willman ${ }^{3}$ \\ ${ }^{1}$ Infrared Processing and Analysis Center, California Institute of Technology, Pasadena, CA 91125, USA; carl@ipac.caltech.edu \\ ${ }^{2}$ Department of Astronomy and Astrophysics, University of Toronto, Toronto, ON M5S 3H4, Canada; hetherington@astro.utoronto.ca, carlberg@astro.utoronto.ca \\ ${ }^{3}$ Department of Physics and Astronomy, Haverford College, Haverford, PA 19041, USA; beth.willman@gmail.com \\ Received 2015 July 16; accepted 2015 September 23; published 2015 October 15
}

\begin{abstract}
Using a shallow, two-color survey carried out with the Dark Energy Camera, we detect the southern, possibly trailing arm of the Orphan Stream. The stream is reliably detected to a decl. of $-38^{\circ}$, bringing the total known length of the Orphan Stream to $108^{\circ}$. We find a slight offset or " $S$ " shape in the stream at $\delta \simeq-14^{\circ}$ that would be consistent with the transition from leading to trailing arms. This coincides with a moderate concentration of $137 \pm$ 25 stars (to $g=21.6$ ) that we consider a possible remnant of the Orphan progenitor. The position of this feature is in agreement with previous predictions.
\end{abstract}

Key words: Galaxy: halo - Galaxy: structure

\section{INTRODUCTION}

The Orphan Stream was among the first stellar debris streams detected in the Sloan Digital Sky Survey (SDSS; Belokurov et al. 2006, 2007; Grillmair 2006). Populous and roughly $2^{\circ}$ wide on the sky, the stream is clearly much broader and stronger than known globular streams such as $\mathrm{Pal} 5$ (Odenkirchen et al. 2003; Grillmair \& Dionatos 2006a). This, along with subsequent findings of a metallicity dispersion of $\sigma[\mathrm{Fe} / \mathrm{H}]=0.56 \mathrm{dex}$ (Casey et al. 2013) and a metallicity gradient amplitude of 0.3 dex (Sesar et al. 2013) led researchers to conclude that the Orphan Stream must be the remnant of a dwarf galaxy. Early modeling efforts suggested that the stream might be related to the neutral hydrogen Complex A (Fellhauer et al. 2007; Jin \& Lynden-Bell 2007) and that the progenitor of the stream might be the nearby dwarf galaxy UMa II (Fellhauer et al. 2007). However, subsequent work by Sales et al. (2008) and Newberg et al. (2010) does not support these ideas.

Newberg et al. (2010) used measured positions and velocities to derive an orbit of the stream and determined that the orbit is prograde, moderately inclined to the Galactic plane $\left(i \approx 34^{\circ}\right)$, fairly eccentric $(e \approx 0.7)$, extending out to $\approx 90 \mathrm{kpc}$ from the Galactic center, and the portion of the stream visible in the SDSS footprint is the leading arm. Based on the rising surface density of the stream at the southern edge of the SDSS footprint (in the direction of decreasing Galactocentric radius and far from apogalacticon), they also suggested that the progenitor would most likely be found between decl. of $0^{\circ}$ and $-16^{\circ}$.

In this Letter, we describe the first results of a shallow imaging survey designed to trace the Orphan Stream well south of the SDSS footprint. We briefly describe the observations in Section 2. We analyze the spatial and color-magnitude characteristics of the stream in Section 3. Concluding remarks are given in Section 4.

\section{OBSERVATIONS}

Using the Orphan orbit estimation of Newberg et al. (2010) as a guide, we imaged a $9^{\circ}-15^{\circ}$ wide swath of sky extending from the celestial equator to $\delta \simeq-53^{\circ}$ and covering an area of $487 \mathrm{deg}^{2}$. This was carried out during just two observing nights using the remarkably efficient Dark Energy Camera (DECam) on the Blanco 4-m telescope at the Cerro Tololo Interamerican Observatory (CTIO). Observations were made in $g$ and $i$, and exposures were kept to two $30 \mathrm{~s}$ dithers per field to maximize the area covered while still reaching well past the mainsequence turnoff of the stream. Observations were carried out over two observing seasons, with one night in 2014 March and another in 2015 March. Conditions were photometric during both nights, with typical seeing of 0 " 9 in $i$ and $1^{\prime \prime}-1$ !" 2 seeing in $g$, though with excursions of $>2^{\prime \prime}$ for a short period during the 2014 run.

The resulting 6.3 TB of data were processed using the 2015 version of the DECam Community Pipeline (Valdes et al. 2013). (2014 data were reprocessed with the 2015 pipeline to take advantage of several improvements.) The data were subsequently transferred to the University of Toronto, where a photometry pipeline based on SExtractor and PSFEx (Bertin \& Arnouts 1996) was constructed to photometer individual images using point-spread function (PSF) fitting.

PSFs, aperture corrections, and second-order color terms were computed for each individual detector. The photometry was calibrated against the SDSS catalog using $\simeq 20 \mathrm{deg}^{2}$ of imaging in the Sloan footprint. Average atmospheric extinction coefficients for CTIO were used throughout. Stars were typically observed at least twice in each filter (with the exception of a small number of stars falling within the CCD gaps), and the individual photometric measurements were combined over all relevant fields and over both observing runs. Within the SDSS footprint, calibration is good to $0.02 \mathrm{mag}$ rms.

Perhaps owing to the variable nature of the PSFs over a field as large as that of DECam, we found that the star/galaxy separation parameter "CLASS_STAR" was rather unreliable, with a spread that varied considerably from the center to the edge of each field. Hence, we relied primarily on the "FWHM_WORLD" and "ELLIPTICITY" parameters to excise sources that were clearly extended. Imposing limits of FWHM_WORLD $<3^{\prime \prime}$, ELLIPTICITY $<0.2$, FLAGS $=0$, and $16<g<21.6$ reduced a catalog of 15 million sources to 3.5 million. The FWHM_WORLD and ELLIPTICITY cuts were deliberately somewhat generous, as tighter constraints resulted in an obvious diminution of source counts from the 
center to the edge of each field. These limits necessarily entail the inclusion of some background galaxies, which will contribute additional noise to the filtered maps, but with a limit of $g=21.6$ this should not be excessive.

Some calibration issues remain unresolved. For example, star counts appear rather more sensitive to airmass than we expect. While many of our fields are essentially complete to $g \approx 23$, others (with airmasses $\geqslant 2$ ) are complete to only $g \approx$ 21.7. These issues will be further explored in a forthcoming contribution. For our present purposes, we avoid these issues by simply cutting off our sample at $g=21.6$.

\section{ANALYSIS}

We used a matched filter to optimally separate the metalpoor stars of the Orphan Stream from the much larger population of foreground disk stars (Rockosi et al. 2002; Grillmair 2009). This technique has been used to detect several streams at surface densities as low as 10 stars deg $^{-2}$ (Grillmair 2006, 2009, 2011; Grillmair \& Dionatos 2006a, 2006b; Bonaca et al. 2012). We generated a filter based on the Padova database of theoretical stellar isochrones (Marigo et al. 2008; Girardi et al. 2010), selecting for stars with $[\mathrm{Fe} / \mathrm{H}]=-1.6$. All stars with $16<g<21.6$ were used, and we dereddened the photometry as a function of position on the sky using the DIRBE/IRAS dust maps of Schlegel et al. (1998), corrected using the prescription of Schlafly \& Finkbeiner (2011). The foreground population was sampled in stream-free regions extending along the edges of our survey area. Figure 1 shows the filtered star count distribution using a filter based on an isochrone with $Z=0.0005$ and an age of $12 \mathrm{Gyr}$, optimized for populations at a distance of $18 \mathrm{kpc}$.

Nearly centered within the survey area is a long, broad feature extending to nearly $-40^{\circ}$. The $18 \mathrm{kpc}$ distance used in Figure 1 corresponds to the strongest stream signal and roughly matches the 19-21 kpc range of distances expected on the basis of an orbit fit to Newberg et al.'s (2010) data compilation for the northern Orphan Stream. Differences may be due to inaccurate matching of the DECam $g$ and $i$ photometry to the Sloan filters assumed by the Padova isochrones, or possibly a metallicity gradient in the Orphan Stream (Sesar et al. 2013). It may also be that $18 \mathrm{kpc}$ is the correct distance of the stream in this region, and that the actual orbit of stream stars in this region needs to be refined.

The northern $10^{\circ}$ of the detected stream matches nicely with the portion of the stream detected in the Sloan footprint. An FWHM of $\approx 1^{\circ} .5-2^{\circ}$ is also consistent with that observed in the northern stream. The stream appears to be reliably detected to $\delta \simeq-38^{\circ}$, below which the character of the distribution changes significantly (see below). This brings the known length of the stream to $\approx 108^{\circ}$. Over the southern interval $-18^{\circ}<\delta<-38^{\circ}$, we find the stream is well fit (to within $0.25)$ by a polynomial of the form:

$$
\alpha=163.147-0.0896 \times \delta+0.00804 \times \delta^{2} .
$$

Figure 2 shows the distribution of $E(B-V)$ over our survey area from the maps of Schlegel et al. (1998). A comparison of Figures 1 and 2 shows that the pattern of the star counts in the region $-39^{\circ}>\delta>-45^{\circ}$ closely matches the filamentary distribution of dust emission and enhanced reddening. Dereddening our photometry has evidently pulled an excess of fainter and redder stars into the sample. Whereas $E(B-V)$ is fairly uniform and ranges from 0.02 to 0.06 over the northern

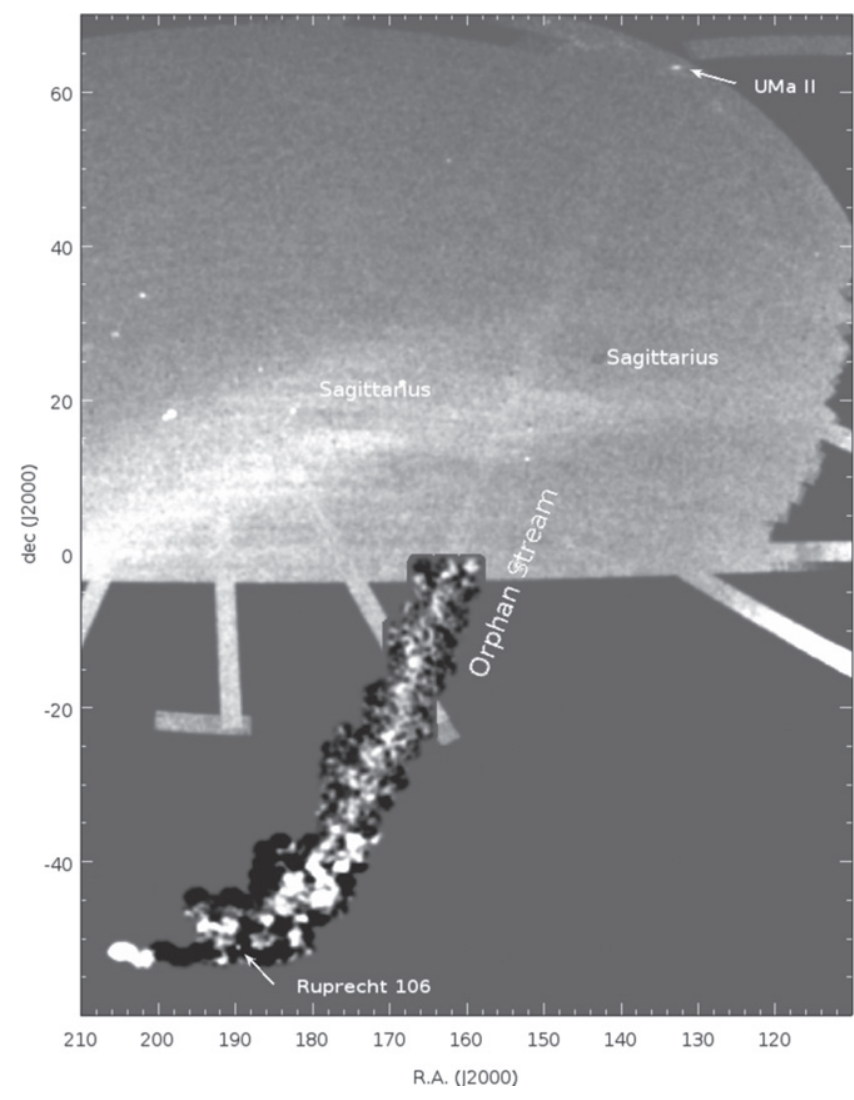

Figure 1. Filtered surface density map of our Decam survey area, overlaid on the SDSS DR10 footprint. The stretch is linear, with lighter areas indicating higher surface densities. The map is the result of a filter based on a Padova isochrone with $[\mathrm{Fe} / \mathrm{H}]=-1.6$, an age of $12 \mathrm{Gyr}$, and shifted to a distance of $18 \mathrm{kpc}$. The Sloan data have been smoothed with a 0.3 Gaussian kernel while the DECam map, owing to its somewhat shallower depth, has been smoothed with a 0.5 kernel. Seeing was $0 !^{\prime \prime} 9-1{ }^{\prime \prime} 8$ over most of the survey area, with two stripes $\left(-37^{\circ}>\delta>-41^{\circ},-45^{\circ}>\delta>-48^{\circ}\right)$ having seeing in excess of $2^{\prime \prime}$. The highest airmasses $(>1.8)$ occurred at $\delta>-7^{\circ}$.

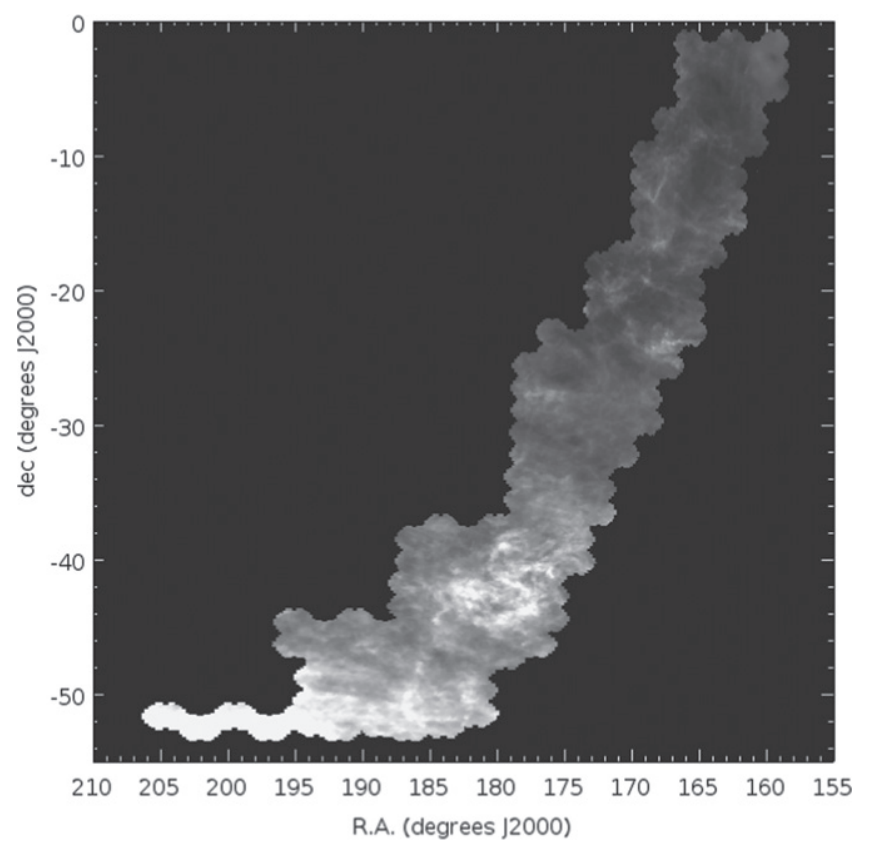

Figure 2. Distribution of $E(B-V)$ over the field shown in Figure 1. Lighter areas indicate higher color excesses. Values of the color excess range from 0.02 in the darkest, northern reaches of the survey to 0.3 in the brightest filaments at $\delta \approx-43^{\circ}$ 


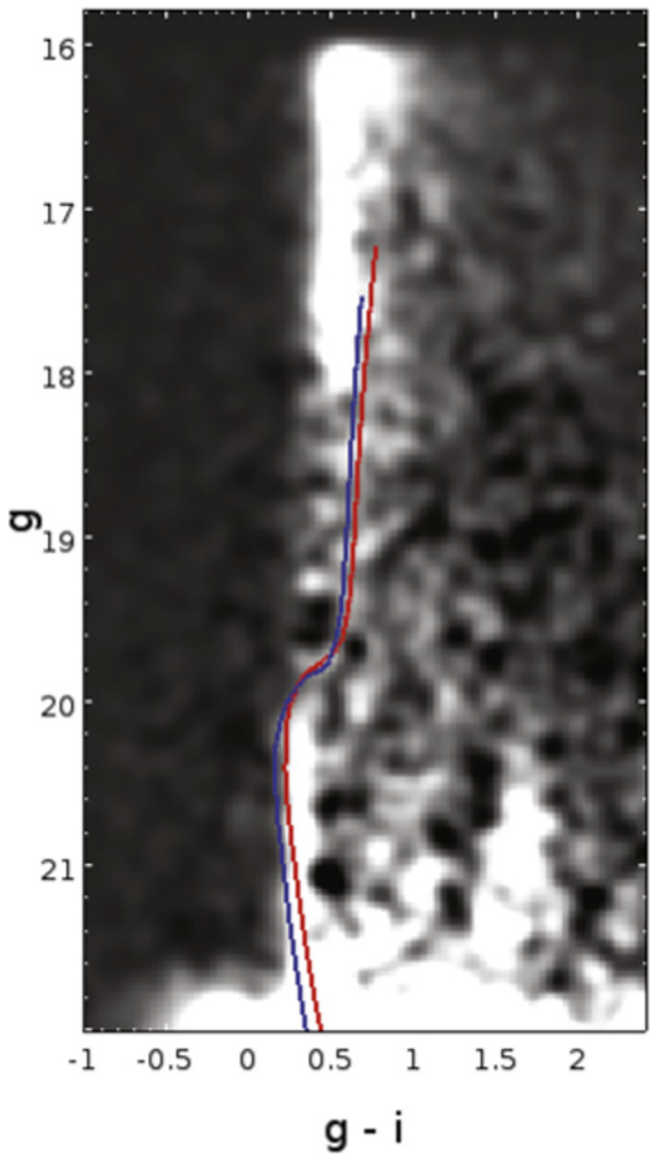

Figure 3. Hess diagram of stars lying within $1^{\circ}$ of the centerline of the Orphan Stream north of $\delta=-36^{\circ}$, after subtraction of the distribution of stars along the edge of the survey area. The result has been convolved with a 0.05 mag Gaussian kernel. Lighter areas indicate higher surface densities. The solid lines shows a Padova isochrone with $[\mathrm{Fe} / \mathrm{H}]=-2.1$ (blue, left) and -1.6 (red right), age $12 \mathrm{Gyr}$, and shifted to distances of 20 and $18 \mathrm{kpc}$, respectively.

half of the survey area, the filamentary structures at $\delta \approx$ $-43^{\circ}$ show color excesses ranging from 0.2 to 0.3 . Arbitrarily scaling down the Schlafly \& Finkbeiner (2011) absorption coefficients reduces the effect, but does not yield any convincing signatures of an underlying stream. Tracing the stream through this region would presumably benefit in the near term from a deep, near-infrared survey, though it should ultimately be detected in Gaia proper motion data.

Figure 3 shows a color-magnitude distribution of stars chosen to lie within $\pm 1^{\circ}$ of the center of the stream north of $\delta=-36^{\circ}$. Overplotted are isochrones for populations with $Z=0.0001([\mathrm{Fe} / \mathrm{H}]=-2.1)$ and $Z=0.0005$ $([\mathrm{Fe} / \mathrm{H}]=-1.6) . Z=0.0005$ appears to match the main sequence somewhat better than $Z=0.0001$, which corresponds to the metallicity found by Newberg et al. (2010) for the blue horizontal branch stars. The value $[\mathrm{Fe} / \mathrm{H}]=-1.6$ used in Figure 1 matches a measurement of $[\mathrm{Fe} / \mathrm{H}]=-1.63$ found by Casey et al. (2013) for red giants. Note that Sesar et al. (2013) see evidence for a metallicity gradient in the northern stream, with the nearer, more southerly stars being $\simeq 0.3$ dex more metal rich than the more northerly, more distant stars.

Figure 4 shows the southern Orphan Stream in greater detail. Overplotted is an orbit fit to the data collected by Newberg et al. (2010) for the northern Orphan Stream. This orbit was computed using the Galactic model of Allen \& Santillan

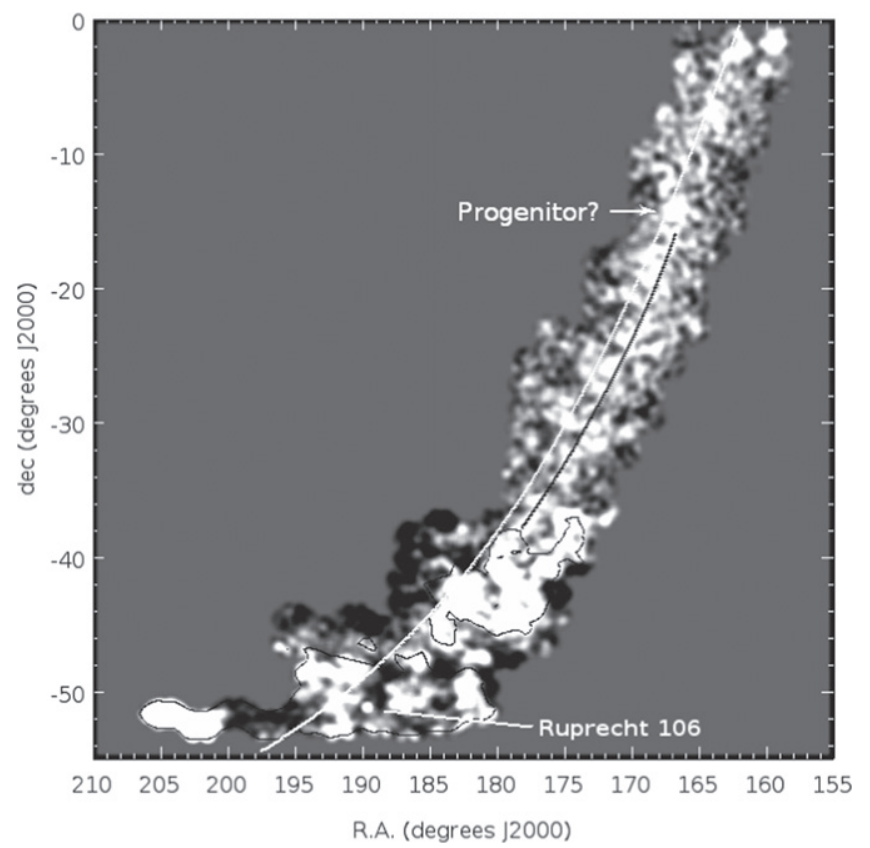

Figure 4. More detailed view of the DECam filtered surface density map in Figure 1 , again smoothed with a Gaussian kernel of width 0.5 . The white curve traces an orbit fit to the position and velocity data of Newberg et al. (2010) for the northern Orphan Stream. The black curve extending to $\delta=-38^{\circ}$ is the fit to the southern portion of the stream given by Equation (1). The black contour in the southern half of the survey area corresponds to $E(B-V)=0.12$ in a smoothed version of Figure 2. Our progenitor candidate is indicated.

(1991), which assumes a spherical halo. The orbit generally matches the trajectory of the southern stream, though offset somewhat toward the east below $\delta \approx-14^{\circ}$. There are a number of possible reasons for the offset: (i) the orbit calculation did not take into account the southern stream (which as of yet has no velocity information), (ii) the effects of halo flattening or triaxiality have not been considered, or (iii) we may be looking at the trailing arm of the stream.

If we use the northern orbit fit as a guide, we see that while it appears to fit the stream reasonably well north of $\delta \approx-14^{\circ}$, an eastward offset of $\approx 1.5$ begins rather suddenly south of $\delta=-14^{\circ}$ and stays roughly constant to $\delta=-38^{\circ}$. At (R.A., decl. $)=\left(167^{\circ},-14^{\circ}\right)$, midway between the northern orbit fit and the run of Equation (1), there is a moderate but significant, 1.5 wide overdensity of stars that is somewhat larger and stronger than the clumps to the immediate north or south. This clump appears to be the extended, northern portion of a feature found by Newberg et al. (2010) in an "outrigger" SEGUE stripe at $\delta \approx-15^{\circ}$. We hypothesize that the transition from the northern to the southern portions of the stream is the "S-shape" signature expected from a progenitor losing stars from its first and second Lagrange points. We further suggest that this clump of stars could be the remnant of the progenitor of the Orphan Stream.

Based on the rise and fall of stream surface density with position along the stream, Newberg et al. (2010) predicted that the progenitor of the Orphan Stream should be situated between $\delta=0^{\circ}$ and $\delta=-16^{\circ}$. This is consistent with the position of our overdensity at $\delta \approx-14^{\circ}$. Moreover, Newberg et al. (2010) determined that the northern portion of the Orphan Stream must be the leading arm. Tidal stripping in a constant- $v_{c}$ potential requires that the leading arm should be made up of stars released from progenitor's first Lagrange point into orbits 
of lower Galactocentric radius $R$. Conversely, the trailing arm will be made up of stars lost from the second Lagrange point, falling behind the progenitor and orbiting at larger $R$. This is consistent with Figure 4; the westward offset of the southern portion of the stream takes it further away from the Galactic center, which is to the left in the figure.

At a distance of $18 \mathrm{kpc}$, a 1.5 offset corresponds to $\approx$ 470 pc. The L1 and L2 lagrange points will always be aligned along a radial to the Galactic center. At the current position of the putative progenitor, we would be viewing it at an angle of $\approx 23^{\circ}$ from the L1-L2 radial. If indeed the northern and southern Orphan Streams are leading and trailing arms, respectively, then the implied physical separation would be $1.2 \mathrm{kpc}$. We consequently take the upper limit on the tidal radius of the progenitor to be $600 \mathrm{pc}$.

The number of stars within the putative progenitor is not large. Examining a square region 1.1 on a side and centered on (R.A., decl. $)=\left(167^{\circ} .125,-14.273\right)$ and comparing with background fields to the east and west, we count stars with $0.16<g-i<0.44$ and $19.9<g<21.6$. Scaling by the area ratios, we find a background-subtracted count of $137 \pm 24$ stars. Integrating over the luminosity function of Omega Cen (de Marchi 1999), we arrive at an approximate total population of $2100 \pm 400$ stars. If this clump is indeed the progenitor of the Orphan Stream, then it would appear to be virtually the last remnant of the original satellite. The surface density of the object is proportional to $r^{-(0.7 \pm 0.3)}$, making it unlikely that the feature could be gravitationally bound.

By definition, the tidal radius $r_{t}^{3}=\left(M_{p} / 2 M_{G}(R)\right) R^{3}$ in a flat rotation curve, where $M_{G}(R)$ is the mass of the Galaxy within Galactocentric radius $R$ and $M_{p}$ is the mass of the progenitor. If we take $R=21 \mathrm{kpc}$ and $M_{G}(R)=1-2 \times 10^{11}$ $M_{\odot}$, we arrive at an upper limit on the progenitor's recent mass of $\sim 4.7-9.3 \times 10^{6} M_{\odot}$. Depending on the number of red giants, the luminosity of the object could range from $1 \times 10^{4}$ to $4 \times 10^{4} L_{\odot}$. If a bound object remains, then $M / L \sim 120-930$ $M_{\odot} / L_{\odot}$

Using the luminosity-metallicity relation of Kirby et al. (2011), the $[\mathrm{Fe} / \mathrm{H}]=-1.6$ measurement of Casey et al. (2013) suggests a total luminosity of the original progenitor of $2.5 \times 10^{6} L_{\odot}$. On the other hand, Newberg et al.'s (2010) value of $[\mathrm{Fe} / \mathrm{H}]=-2.1$ implies $6 \times 10^{5} L_{\odot}$. Our luminosity estimate above would suggest that the progenitor has lost between $94 \%$ and $100 \%$ of its original mass.

There are other surface density peaks evident in Figure 4, but we are less inclined to consider these as progenitor candidates as they do not show the morphological indicators (e.g., offsets) we would associate with the transition from leading to trailing arms. Given the orientation of the Orphan Stream and our view of it, such a feature should be readily apparent.

We note also that near the southernmost end of the survey area is the globular cluster Ruprecht 106. This cluster is situated along the plausible extension of the Orphan Stream. However, while its metallicity of $[\mathrm{Fe} / \mathrm{H}]=-1.67$ (Harris 1996) is similar to that of the Orphan Stream, its distance of $12 \mathrm{kpc}$ and radial velocity of $-44 \mathrm{~km} \mathrm{~s}^{-1}$ are at odds with values of $21 \mathrm{kpc}$ and $+72 \mathrm{~km} \mathrm{~s}^{-1}$ predicted by the orbit fit to the northern Orphan Stream. We conclude that Ruprecht 106 is unlikely to be physically associated with the stream.

\section{CONCLUSIONS}

Using a large, shallow DECam survey, we have traced the Orphan Stream from the celestial equator to $\delta \simeq-38^{\circ}$. The stream appears to be roughly $18 \mathrm{kpc}$ distant, and its trajectory generally agrees with expectations based on orbit fits to the northern stream. The color-magnitude distribution is clearly metal poor and appears similar to that of the northern Orphan Stream. We find a stellar concentration and apparent offsets in the stream that would be consistent with a remnant progenitor.

This southern extension of the Orphan Stream should enable significant improvements in constraining the overall orbit and, ultimately, the shape of the Galactic potential. This is particularly interesting in that the Orphan Stream passes through quadrants of the halo not probed by the Sagittarius stream. Slightly deeper than the present survey, the PanSTARRS survey may enable us to improve the signal-to-noise ratio somewhat for $\delta>-30^{\circ}$. For more southerly regions, where we are strongly affected by reddening, a deep, nearinfrared survey may help to trace the stream still further south.

We gratefully acknowledge Jonathan Hargis for helpful suggestions in the course of developing our photometry pipeline. This project used data obtained with the Dark Energy Camera (DECam), which was constructed by the Dark Energy Survey (DES) collaboration. Funding for the DES Projects has been provided by the DOE and NSF(USA), MISE(Spain), STFC(UK), HEFCE(UK). NCSA(UIUC), KICP(U. Chicago), CCAPP(Ohio State), MIFPA(Texas A\&M), CNPQ, FAPERJ, FINEP (Brazil), MINECO(Spain), DFG(Germany), and the collaborating institutions in the Dark Energy Survey, which are Argonne Lab, UC Santa Cruz, University of Cambridge, CIEMAT-Madrid, University of Chicago, University College London, DES-Brazil Consortium, University of Edinburgh, ETH Zurich, Fermilab, University of Illinois, ICE (IEECCSIC), IFAE Barcelona, Lawrence Berkeley Lab, LMU Munchen and the associated Excellence Cluster Universe, University of Michigan, NOAO, University of Nottingham, Ohio State University, University of Pennsylvania, University of Portsmouth, SLAC National Lab, Stanford University, University of Sussex, and Texas A\&M University.

Facility: CTIO:Blanco (DECam).

\section{REFERENCES}

Allen, C., \& Santillan, A. 1991, RMxAA, 22, 255

Belokurov, V., Evans, N. W., Irwin, M. J., et al. 2007, ApJ, 658, 337

Belokurov, V., Zucker, D. B., Evans, N. W., et al. 2006, ApJ, 642, 137

Bertin, E., \& Arnouts, S. 1996, A\&AS, 117, 393

Bonaca, A., Geha, M., \& Kallivayalil, N. 2012, ApJ, 760, 6

Casey, A. R., Da Costa, G., Keller, S. C., \& Maunder, E. 2013, ApJ, 764, 39 de Marchi, G. 1999, AJ, 117, 303

Fellhauer, M., Evans, N. W., Belokurov, V., et al. 2007, MNRAS, 375, 1171 Girardi, L., Williams, B. F., Gilbert, K. M., et al. 2010, ApJ, 724, 1030

Grillmair, C. J. 2006, ApJ, 645, 37

Grillmair, C. J. 2009, ApJ, 693, 1118

Grillmair, C. J. 2011, ApJ, 738, 98

Grillmair, C. J., \& Dionatos, O. 2006a, ApJ, 641, 37

Grillmair, C. J., \& Dionatos, O. 2006b, ApJ, 643, 17

Harris, W. E. 1996, AJ, 112, 1487

Jin, S., \& Lynden-Bell, D. 2007, MNRAS, 378, 64

Kirby, E. N., Lanfranchi, G. A., Simon, J. D., Cohen, J. G., \& Guhathakurta, P. 2011, ApJ, 727, 78

Küpper, A. H. W., Lane, R. R., \& Heggie, D. C. 2012, MNRAS, 420, 2700

Marigo, P., Girardi, L., Bressan, A., et al. 2008, A\&A, 482, 883

Newberg, H. J., Willett, B. A., Yanny, B., \& Xu, Y. 2010, ApJ, 711, 32

Odenkirchen, M., Grebel, E. K., Dehnen, W., et al. 2003, AJ, 126, 2385 
Rockosi, C. M., Odenkirchen, M., Grebel, E. K., et al. 2002, AJ, 124, 349

Sales, L. V., Helmi, A., Starkenburg, E., et al. 2008, MNRAS, 389, 1391

Schlafly, E. F., \& Finkbeiner, D. P. 2011, ApJ, 737, 103

Schlegel, D. J., Finkbeiner, D. P., \& Davis, M. 1998, ApJ, 500, 525
Sesar, B., Grillmair, C. J., Cohen, J. G., et al. 2013, ApJ, 776, 26

Valdes, F., Gruendl, R., \& the DES Project 2013, in ASP Conf. Ser. 485, Astronmomical Data Analysis Software and Systems XXIII (San Francisco, CA: ASP), 379 